\title{
Higher moments of primes in short intervals II
}

\author{
Tsz Ho Chan
}

July 31, 2018

\begin{abstract}
Given good knowledge on the even moments, we derive asymptotic formulas for $\lambda$-th moments of primes in short intervals and prove "equivalence" result on odd moments. We also provide numerical evidence in support of these results.
\end{abstract}

\section{Introduction}

In [5, Montgomery and Soundararajan studied the moments

$$
M_{k}(X ; h):=\int_{1}^{X}(\psi(x+h)-\psi(x)-h)^{k} d x
$$

where $k$ is a positive integer, $\psi(x)=\sum_{n \leq x} \Lambda(n)$ and $\Lambda(n)$ is von Mangoldt lambda function. They proved that, under a strong form of Hardy-Littlewood prime- $k$ tuple conjecture, for small $\epsilon>0$, there is a $\epsilon^{\prime}>0$,

$$
M_{k}(X ; h)=\mu_{k} h^{k / 2} \int_{1}^{X}\left(\log \frac{x}{h}+B\right)^{k / 2} d x+O_{k}\left(h^{k / 2} X^{1-\epsilon}\right)
$$

uniformly for $(\log X)^{15 k^{2}} \leq h \leq X^{1 / k-\epsilon^{\prime}}$ where $\mu_{k}=1 \cdot 3 \cdots(k-1)$ if $k$ is even, and $\mu_{k}=0$ if $k$ is odd. Here $B=1-C_{0}-\log 2 \pi$ and $C_{0}$ denotes Euler's constant. One further expects that (10) holds uniformly for $X^{\epsilon^{\prime}} \leq h \leq X^{1-\epsilon^{\prime}}$. Consider the closely related moments:

$$
\widetilde{M}_{k}(X ; \delta):=\int_{1}^{X}(\psi(x+\delta x)-\psi(x)-\delta x)^{k} d x .
$$

In 19, the author proved that, roughly speaking, (1) holding uniformly for $X^{\epsilon^{\prime}} \leq$ $h \leq X^{1-\epsilon^{\prime}}$ is equivalent to

$$
\widetilde{M}_{k}(X ; \delta)=\frac{\mu_{k}}{\frac{k}{2}+1} X^{k / 2+1} \delta^{k / 2}\left(\log \frac{1}{\delta}+B\right)^{k / 2}+O_{k}\left(\delta^{k / 2} X^{k / 2+1-\epsilon}\right)
$$

holding uniformly for $X^{-1+\epsilon^{\prime}} \leq \delta \leq X^{-\epsilon^{\prime}}$. 
In this article, we shall study the following more general moments: For $\lambda>0$,

$$
\begin{aligned}
& M_{\lambda}(X ; h):=\int_{1}^{X}|\psi(x+h)-\psi(x)-h|^{\lambda} d x, \\
& \widetilde{M}_{\lambda}(X ; \delta):=\int_{1}^{X}|\psi(x+\delta x)-\psi(x)-\delta x|^{\lambda} d x .
\end{aligned}
$$

Instead of (11) and (3), we can assume the following weaker versions: For some $\epsilon>0$ and all even positive integer $k$,

$$
\begin{aligned}
\int_{1}^{X}(\psi(x+h)-\psi(x)-h)^{k} d x= & \frac{\Gamma(k+1)}{\Gamma\left(\frac{k}{2}+1\right) 2^{k / 2}} X h^{k / 2}\left(\log \frac{X}{h}\right)^{k / 2} \\
& +O\left(A^{B^{k}} X h^{k / 2} \log ^{k / 2-1} X\right)
\end{aligned}
$$

for $X^{\epsilon} \leq h \leq X^{1-\epsilon}$, and

$$
\begin{aligned}
\int_{1}^{X}(\psi(x+\delta x)-\psi(x)-\delta x)^{k} d x= & \frac{\Gamma(k+1)}{\Gamma\left(\frac{k}{2}+2\right) 2^{k / 2}} X^{k / 2+1} \delta^{k / 2}\left(\log \frac{1}{\delta}\right)^{k / 2} \\
& +O\left(A^{B^{k}} X^{k / 2+1} \delta^{k / 2} \log ^{k / 2-1} X\right)
\end{aligned}
$$

for $X^{-1+\epsilon} \leq \delta \leq X^{-\epsilon}$. Remarks: $1 . \Gamma(x)$ is the gamma function $(\Gamma(n+1)=n$ ! for non-negative integer $n$ ). 2. The weaker versions suffice because our proof of the following theorems gives such poor error terms that only the first main terms matter. 3. We make the implicit constants' dependence on $k$ explicit in the error terms. Here $A, B$ are some absolute constants greater than 1 and $A^{B^{k}}$ is the result of tracing the $k$ dependency of the error terms in [6] and explicitly. 4. $A$ and $B$ may depend on $\epsilon$ but we treat $\epsilon$ as fixed to start with.

We shall prove the following

Theorem 1.1. If (6) is true for every even positive integer $k$ in $X^{\epsilon} \leq h \leq X^{1-\epsilon}$, then for any $\lambda>0$,

$$
\begin{aligned}
\int_{1}^{X}|\psi(x+h)-\psi(x)-h|^{\lambda} d x & =\frac{\Gamma(\lambda+1)}{\Gamma\left(\frac{\lambda}{2}+1\right) 2^{\lambda / 2}} X h^{\lambda / 2}\left(\log \frac{X}{h}\right)^{\lambda / 2} \\
& +O_{\lambda}\left(\frac{X h^{\lambda / 2}\left(\log \frac{X}{h}\right)^{\lambda / 2}}{\log _{4} X}\right)
\end{aligned}
$$

for $X^{\epsilon} \leq h \leq X^{1-\epsilon}$. Here $\log _{n} x$ stands for $n$ times iterated logarithm $\left(\log _{1} x=\right.$ $\log x$ and $\left.\log _{i+1} x=\log \log _{i} x\right)$.

Theorem 1.2. If (7) is true for every even positive integer $k$ in $X^{-1+\epsilon} \leq \delta \leq$ $X^{-\epsilon}$, then for any $\lambda>0$,

$$
\begin{aligned}
\int_{1}^{X}|\psi(x+\delta x)-\psi(x)-\delta x|^{\lambda} d x & =\frac{\Gamma(\lambda+1)}{\Gamma\left(\frac{\lambda}{2}+2\right) 2^{\lambda / 2}} X^{\lambda / 2+1} \delta^{\lambda / 2}\left(\log \frac{1}{\delta}\right)^{\lambda / 2} \\
& +O_{\lambda}\left(\frac{X^{\lambda / 2+1} \delta^{\lambda / 2}\left(\log \frac{1}{\delta}\right)^{\lambda / 2}}{\log _{4} X}\right)
\end{aligned}
$$


for $X^{-1+\epsilon} \leq \delta \leq X^{-\epsilon}$.

Using these theorems, we shall prove "equivalence" result in section 3 . We shall give numerical evidence in the last section. This work is part of the author's $2002 \mathrm{PhD}$ thesis.

\section{Proof of Theorem 1.1 and 1.2}

We need Legendre's double formula for gamma function:

$$
\sqrt{\pi} \Gamma(2 z)=2^{2 z-1} \Gamma(z) \Gamma\left(z+\frac{1}{2}\right) .
$$

Since the proof of Theorem 1.1 and Theorem 1.2 are very similar, we shall give the proof of Theorem 1.1 only.

Proof of Theorem 1.1 The method is essentially that of Ghosh [2] and 3]. There are two cases depending on the size of $\lambda$ :

(i) $0<\lambda \leq 1$.

Let $F(x)=\frac{\psi(x+h)-\psi(x)-h}{2^{-1 / 2} h^{1 / 2}(\log X / h)^{1 / 2}}$ and $G_{\lambda}=\int_{0}^{\infty} \frac{(\sin u)^{2}}{u^{1+\lambda}} d u$. For any $\mu \geq 1$,

$$
\begin{aligned}
|F(x)|^{\lambda} & =\frac{1}{G_{\lambda}} \int_{0}^{\infty} \frac{(\sin |F(x)| u)^{2}}{u^{1+\lambda}} d u \\
& =\frac{1}{G_{\lambda}} \int_{0}^{\mu} \frac{(\sin |F(x)| u)^{2}}{u^{1+\lambda}} d u+O\left(\frac{1}{\lambda G_{\lambda}} \mu^{-\lambda}\right) .
\end{aligned}
$$

Hence,

$$
\int_{1}^{X}|F(x)|^{\lambda} d x=\frac{1}{G_{\lambda}} \int_{0}^{\mu} \int_{1}^{X} \frac{(\sin |F(x)| u)^{2}}{u^{1+\lambda}} d x d u+O_{\lambda}\left(\frac{X}{\mu^{\lambda}}\right) .
$$

Note that $(\sin x)^{2}=\frac{1}{2} \sum_{j=1}^{N} \frac{(-1)^{j+1}(2 x)^{2 j}}{(2 j) !}+O\left(\frac{(2 x)^{2 N+2}}{(2 N+2) !}\right)$. So the main term of (9) is

$$
\begin{aligned}
& \quad \frac{1}{2 G_{\lambda}} \int_{0}^{\mu} \frac{1}{u^{1+\lambda}} \sum_{j=1}^{N} \frac{(-1)^{j+1}(2 u)^{2 j}}{(2 j) !}\left(\int_{1}^{X}|F(x)|^{2 j} d x\right) d u \\
& +O\left(\frac{4^{N}}{(2 N+2) !} \int_{0}^{\mu} u^{2 N} d u \int_{1}^{X}|F(x)|^{2 N+2} d x\right) .
\end{aligned}
$$

Using assumption (6), the error term in (10) is bounded by

$$
\frac{(2 \mu)^{2 N}}{(N+2) !} X \mu+\frac{A^{B^{N}}(2 \mu)^{2 N}}{(2 N+3) !} \frac{X \mu}{\log X} .
$$


Using (6), the main term of (10) contributes

$\frac{X}{2 G_{\lambda}} \int_{0}^{\mu} \frac{1}{u^{1+\lambda}} \sum_{j=1}^{N} \frac{(-1)^{j+1}}{(2 j) !} \frac{\Gamma(2 j+1)}{\Gamma(j+1)}(2 u)^{2 j} d u+O\left(\frac{X}{\log X} \sum_{j=1}^{N} \frac{A^{B^{j}}}{(2 j) !} \int_{0}^{\mu} u^{2 j-2} d u\right)$.

The above error term is bounded by

$$
\frac{X}{\log X} A^{B^{N}} \sum_{j=1}^{N} \frac{\mu^{2 j-1}}{(2 j) !} \ll \frac{X}{\log X} A^{B^{N}} e^{\mu} .
$$

Using (8) with $z=j+\frac{1}{2}$, the main term of (12) becomes

$$
\begin{aligned}
& \frac{X}{2 G_{\lambda}} \int_{0}^{\mu} \frac{1}{u^{1+\lambda}} \sum_{j=1}^{N} \frac{(-1)^{j+1}}{(2 j) !} \frac{2^{2 j}}{\sqrt{\pi}} \Gamma\left(j+\frac{1}{2}\right)(2 u)^{2 j} d u \\
= & \frac{X}{2 \sqrt{\pi} G_{\lambda}} \int_{0}^{\mu} \frac{1}{u^{1+\lambda}} \sum_{j=1}^{N} \frac{(-1)^{j+1}}{(2 j) !}(4 u)^{2 j}\left(\int_{0}^{\infty} x^{j-1 / 2} e^{-x} d x\right) d u \\
= & \frac{X}{\sqrt{\pi} G_{\lambda}} \int_{0}^{\infty} x^{-1 / 2} e^{-x} \int_{0}^{\mu} \frac{1}{u^{1+\lambda}}\left((\sin 2 \sqrt{x} u)^{2}+O\left(\frac{(4 \sqrt{x} u)^{2 N+2}}{(2 N+2) !}\right)\right) d u d x .
\end{aligned}
$$

The contribution from the above error term is

$$
\ll_{\lambda} \frac{4^{2 N+2}(N+1) !}{(2 N+2) !} \mu^{2 N+2-\lambda} X
$$

The contribution from the main term is

$$
\begin{aligned}
& =\frac{X}{\sqrt{\pi} G_{\lambda}} \int_{0}^{\infty} x^{-1 / 2} e^{-x}\left(\int_{0}^{\infty} \frac{(\sin 2 \sqrt{x} u)^{2}}{u^{1+\lambda}} d u+O\left(\frac{1}{\lambda \mu^{\lambda}}\right)\right) d x \\
& =\frac{2^{\lambda}}{\sqrt{\pi}} \Gamma\left(\frac{\lambda+1}{2}\right) X+O_{\lambda}\left(\frac{X}{\mu^{\lambda}}\right) \\
& =\frac{\Gamma(\lambda+1)}{\Gamma\left(\frac{\lambda}{2}+1\right)} X+O_{\lambda}\left(\frac{X}{\mu^{\lambda}}\right)
\end{aligned}
$$

by the definition of $G_{\lambda}$ and (8). Combining this with (9), (10), (11), (12), (13) and (14), we have

$$
\int_{1}^{X}|F(x)|^{\lambda} d x=\frac{\Gamma(\lambda+1)}{\Gamma\left(\frac{\lambda}{2}+1\right)} X+\text { error }
$$

where

$$
\text { error } \ll_{\lambda} \frac{X}{\mu^{\lambda}}+\frac{(2 \mu)^{2 N+1}}{(N+1) !} X+\frac{A^{B^{N}}(2 \mu)^{2 N+2}}{(2 N+3) !} \frac{X}{\log X}+A^{B^{N}} e^{\mu} \frac{X}{\log X} .
$$


Now, we choose $N=\frac{\log _{3} X}{\log _{4} X}$ and $\mu=\sqrt[\lambda]{\log _{4} X}$, then one can check that $A^{B^{N}} \ll$ $\sqrt{\log X}, e^{\mu} \ll_{\lambda}(\log X)^{\frac{1}{4}}$, and using Stirling's formula,

$$
\begin{aligned}
\frac{(2 \mu)^{2 N+2}}{(N+1) !} & \ll \frac{e^{(2 N+2) \log 2 \mu}}{\sqrt{N}(N / e)^{N}} \ll \frac{1}{\sqrt{N}} e^{(2 N+2) \log 2 \mu-N \log (N / e)} \\
& \ll_{\lambda} \quad \frac{1}{\sqrt{N}} \ll \frac{1}{\log _{3} X} .
\end{aligned}
$$

Consequently, (15) becomes

$$
\int_{1}^{X}|F(x)|^{\lambda} d x=\frac{\Gamma(\lambda+1)}{\Gamma\left(\frac{\lambda}{2}+1\right)} X+O_{\lambda}\left(\frac{X}{\log _{4} X}\right)
$$

which gives the theorem for $0<\lambda \leq 1$ after multiplying through by $\left(\frac{1}{\sqrt{2}} h^{1 / 2}\left(\log \frac{X}{h}\right)^{1 / 2}\right)^{\lambda}$.

(ii) $1<\lambda$.

Let $\lambda=2 m+1+\theta$ where $m$ is a non-negative integer and $0<\theta \leq 2$. Let $D_{\theta}=\int_{0}^{\infty} \frac{(\sin u)^{4}}{u^{2+\theta}} d u$. Since

$$
\begin{aligned}
|F(x)|^{\lambda} & =\frac{|F(x)|^{2 m}}{D_{\theta}} \int_{0}^{\infty} \frac{(\sin |F(x)| u)^{4}}{u^{2+\theta}} d u \\
& =\frac{|F(x)|^{2 m}}{D_{\theta}} \int_{0}^{Y} \frac{(\sin |F(x)| u)^{4}}{u^{2+\theta}} d u+O_{\lambda}\left(|F(x)|^{2 m} Y^{-1-\theta}\right) .
\end{aligned}
$$

Then, similar to the calculation in case (i), for some $Y \geq 1$,

$$
\int_{1}^{X}|F(x)|^{\lambda} d x=\frac{1}{D_{\theta}} \int_{0}^{Y} \frac{1}{u^{2+\theta}}\left(\int_{1}^{X}|F(x)|^{2 m}(\sin |F(x)| u)^{4} d x\right) d u+O_{\lambda}\left(Y^{-1-\theta} X\right)
$$

by [6]). From (7) of Ghosh [3], we have

$$
(\sin u)^{4}=\frac{1}{8} \sum_{j=2}^{N} b_{j} u^{2 j}+O\left(\frac{(4 u)^{2 N+2}}{(2 N+2) !}\right) \text { where } b_{j}=\frac{(-4)^{j+1}}{(2 j) !}\left(4^{j-1}-1\right),
$$

and $N$ is an integer, exceeding 2 , which will be chosen later. Using this Taylor series and (6), the main term of (16) equals

$$
\begin{gathered}
\frac{X}{8 D_{\theta}} \int_{0}^{Y} \frac{1}{u^{2+\theta}} \sum_{j=2}^{N} b_{j} u^{2 j} \frac{\Gamma(2(m+j)+1)}{\Gamma((m+j)+1)} d u \\
\left.+O\left(\frac{X}{\log X} \sum_{j=2}^{N} A^{B^{j}}\left|b_{j}\right| \int_{0}^{Y} u^{2 j-2-\theta} d u\right)+\int_{1}^{X}|F(x)|^{2 m} \frac{(4|F(x)|)^{2 N+2}}{(2 N+2) !} \int_{0}^{Y} u^{2 N-\theta} d u d x\right) .
\end{gathered}
$$

The error term of (17) is

$$
\ll \frac{A^{B^{N}} e^{4 Y}}{Y^{1+\theta}} \frac{X}{\log X}+\frac{4^{2 N+2} Y^{2 N+1}}{(2 N+2) !}\left(\frac{(2(m+N+1)) !}{(m+N+2) !}+\frac{A^{B^{m+N+1}}}{\log X}\right) X .
$$


By (8), the main term of (17)

$$
\begin{aligned}
& =\frac{X}{D_{\theta}} \int_{0}^{Y} \frac{1}{u^{2+\theta}} \sum_{j=2}^{N} \frac{b_{j}}{8} u^{2 j} \frac{2^{2(m+j)}}{\sqrt{\pi}}\left(\int_{0}^{\infty} x^{m+j-1 / 2} e^{-x} d x\right) d u \\
& =\frac{2^{2 m} X}{\sqrt{\pi} D_{\theta}} \int_{0}^{\infty} x^{m-1 / 2} e^{-x} \int_{0}^{Y} \frac{1}{u^{2+\theta}}\left((\sin 2 \sqrt{x} u)^{4}+O\left(\frac{(8 \sqrt{x} u)^{2 N+2}}{(2 N+2) !}\right)\right) d u d x .
\end{aligned}
$$

Contribution from the error is

$$
\ll_{\lambda} \frac{(N+m+1) !}{(2 N+2) !} 2^{4 N} Y^{2 N+1} X
$$

while the main term

$$
\begin{aligned}
& =\frac{2^{2 m} X}{\sqrt{\pi} D_{\theta}} \int_{0}^{\infty} x^{m-1 / 2} e^{-x}\left(\int_{0}^{\infty} \frac{(\sin 2 \sqrt{x} u)^{4}}{u^{2+\theta}} d u+O\left(Y^{-1-\theta}\right)\right) d x \\
& =\frac{2^{2 m+1+\theta}}{\sqrt{\pi}} X \int_{0}^{\infty} x^{m+\theta / 2} e^{-x} d x+O_{\lambda}\left(X Y^{-1-\theta}\right) \\
& =\frac{\Gamma(\lambda+1)}{\Gamma\left(\frac{\lambda}{2}+1\right)} X+O_{\lambda}\left(X Y^{-1-\theta}\right)
\end{aligned}
$$

by (8). Therefore, combining this with (16), (17), (18) and (19), we have

$$
\int_{1}^{X}|F(x)|^{\lambda} d x=\frac{\Gamma(\lambda+1)}{\Gamma\left(\frac{\lambda}{2}+1\right)} X+\text { error }
$$

where

$$
\begin{aligned}
\text { error } & \ll_{\lambda} X\left(\frac{1}{Y}+\frac{A^{B^{N}} e^{4 Y}}{Y \log X}+4^{2 N} Y^{2 N+1} \frac{(2(m+N+1)) !}{(2 N+2) !(m+N+2) !}\right. \\
& \left.+\frac{4^{2 N} A^{B^{N}}}{(2 N+2) !} \frac{Y^{2 N+1}}{\log X}+\frac{4^{2 N}(N+m+1) !}{(2 N+2) !} Y^{2 N+1}\right) .
\end{aligned}
$$

By Stirling's formula, 211

$$
\ll_{\lambda} X\left(\frac{1}{Y}+\frac{A^{B^{N}} e^{4 Y}}{Y \log X}+\frac{4^{4 N}}{(N+1)^{N-m}} Y^{2 N+1}+\frac{A^{B^{N}}}{(N+1)^{2 N+2}} \frac{Y^{2 N+1}}{\log X}\right) .
$$

Now, pick $N=\frac{\log _{3} X}{\log _{4} X}$ and $Y=\sqrt{\log _{3} X}$, we have $A^{B^{N}} \ll \sqrt{\log X}, 4^{4 N} \ll$ $\sqrt[4]{\log \log X},(N+1)^{N-m} \gg_{\lambda} \log \log X, e^{4 Y} \ll \log \log X$ and $Y^{2 N+1} \ll \sqrt{\log \log X}$. Thus, (21) is $O_{\lambda}\left(\frac{X}{\sqrt[3]{\log _{3} X}}\right)$ and we get the theorem after multiplying (20) by $\left(\frac{1}{\sqrt{2}} h^{1 / 2}\left(\log \frac{X}{h}\right)^{1 / 2}\right)^{\lambda}$. 


\section{3 "Equivalence" for odd moments}

In [1, the author proved that (1) and (3) are roughly equivalent to one another when $k$ is even. One would like to prove a similar statement when $k$ is odd. However, the difficulty lies in that we no longer have asymptotic formulas. But, if one has good knowledge about all the even moments then it is possible to get the following

Theorem 3.1. Assume Riemann Hypothesis. If [6) holds in $X^{\epsilon} \leq h \leq X^{1-\epsilon}$ for some $\epsilon>0$ and all positive even integer $k$, then, for any positive odd integer $n$,

$$
\int_{1}^{X}(\psi(x+h)-\psi(x)-h)^{n} d x=o\left(X h^{n / 2}(\log X)^{n / 2}\right)
$$

for $X^{\epsilon} \leq h \leq X^{1-\epsilon}$ implies that, for some $\epsilon_{1}>0$,

$$
\int_{1}^{X}(\psi(x+\delta x)-\psi(x)-\delta x)^{n} d x=o\left(X^{n / 2+1} \delta^{n / 2}\left(\log \frac{1}{\delta}\right)^{n / 2}\right)
$$

for $X^{-1+2 \epsilon+2 \epsilon_{1}} \leq \delta \leq X^{-\epsilon} / 2$.

Conversely, one also has

Theorem 3.2. Assume Riemann Hypothesis. If (7) holds in $X^{-1+\epsilon} \leq \delta \leq X^{-\epsilon}$ for some $\epsilon>0$ and all positive even integer $k$, then, for any positive odd integer $n$,

$$
\int_{1}^{X}(\psi(x+\delta x)-\psi(x)-\delta x)^{n} d x=o\left(X^{n / 2+1} \delta^{n / 2}\left(\log \frac{1}{\delta}\right)^{n / 2}\right)
$$

for $X^{-1+\epsilon} \leq \delta \leq X^{-\epsilon}$ implies that, for some $\epsilon_{1}>0$,

$$
\int_{1}^{X}(\psi(x+h)-\psi(x)-h)^{n} d x=o\left(X h^{n / 2}(\log X)^{n / 2}\right)
$$

for $X^{2 \epsilon+\epsilon_{1}} \leq h \leq X^{1-(n / 2+1) \epsilon-2 \epsilon_{1}} / 2$.

Remarks: 1 . The proofs of the above theorems are very similar to the proofs of theorems in [1. We shall give a sketch for Theorem 3.1 only. 2. We did not optimize the ranges for $h$ and $\delta$ in the above theorems. Improvements are possible since the error terms in the proofs in 1 are smaller.

Sketch of proof of Theorem 3.1 Observe that

$$
\begin{aligned}
& \int_{1}^{X}|\psi(x+h)-\psi(x)-h|^{n} d x+\int_{1}^{X}(\psi(x+h)-\psi(x)-h)^{n} d x \\
= & 2 \int_{1}^{X} \max \{\psi(x+h)-\psi(x)-h, 0\}^{n} d x .
\end{aligned}
$$


Thus, by the assumptions in Theorem 3.1 we can apply Theorem 1.1 and get

$$
\begin{aligned}
\int_{1}^{X} \max \{\psi(x+h)-\psi(x)-h, 0\}^{n} d x & =\frac{1}{2} \frac{\Gamma(n+1)}{\Gamma\left(\frac{n}{2}+1\right) 2^{n / 2}} X h^{n / 2}\left(\log \frac{X}{h}\right)^{n / 2} \\
& +o\left(X h^{n / 2}(\log X)^{n / 2}\right)
\end{aligned}
$$

Now, for any $0<\epsilon_{1}<\epsilon$, one can imitate Saffari and Vaughan's argument as in Theorem 3.1 of [1] and get

$$
\begin{aligned}
& \int_{0}^{\Delta} \int_{1}^{X} \max \{\psi(x+\delta x)-\psi(x)-\delta x, 0\}^{n} d x d \delta \\
= & \frac{\Gamma(n+1)}{2 \Gamma\left(\frac{n}{2}+2\right) 2^{n / 2}} \int_{0}^{\Delta X} h^{n / 2}\left(\log \frac{X}{h}\right)^{n / 2} d h+o\left(\Delta^{n / 2+1} X^{n / 2+1}\left(\log \frac{1}{\Delta}\right)^{n / 2}\right)
\end{aligned}
$$

for $X^{-1+2 \epsilon+\epsilon_{1}} \leq \delta \leq X^{-\epsilon}$. Let $f(x, h)=\max \{\psi(x+h)-\psi(x)-h, 0\}, g(x, \delta x)=$ $f(x, \Delta x)$ for $\Delta \leq \delta \leq(1+\eta) \Delta$. Following the argument in Theorem 3.1 of [1] without choosing $\eta$ explicitly or simply following the argument in 4, one has, for $X^{-1+2 \epsilon+2 \epsilon_{1}} \leq \Delta \leq X^{-\epsilon} / 2$,

$$
\begin{aligned}
& \int_{1}^{X} \max \{\psi(x+\Delta x)-\psi(x)-\Delta x, 0\}^{n} d x \\
= & \frac{1}{2} \frac{\Gamma(n+1)}{\Gamma\left(\frac{n}{2}+2\right) 2^{n / 2}} X^{n / 2+1} \Delta^{n / 2}\left(\log \frac{1}{\Delta}\right)^{n / 2}+o\left(X^{n / 2+1} \Delta^{n / 2}\left(\log \frac{1}{\Delta}\right)^{n / 2}\right)
\end{aligned}
$$

by letting $\eta$ approach 0 sufficiently slowly. The only difference in the argument is the use of

$$
\begin{aligned}
|f(x, \delta x)-g(x, \delta x)| & \leq|(\psi(x+\delta x)-\psi(x)-\delta x)-(\psi(x+\Delta x)-\psi(x)-\Delta x)| \\
& =|\psi((x+\Delta x)+(\delta-\Delta) x)-(\delta-\Delta) x|
\end{aligned}
$$

for $\Delta \leq \delta \leq(1+\eta) \Delta$ when one estimates the integral $\iint|f-g|^{n}$. The above is justified by

$$
|\max \{a, 0\}-\max \{b, 0\}| \leq|a-b|
$$

which can be easily verified by considering different cases of signs for $a$ and $b$. Similarly, one has

$$
\begin{aligned}
& \int_{1}^{X} \min \{\psi(x+\Delta x)-\psi(x)-\Delta x, 0\}^{n} d x \\
= & -\frac{1}{2} \frac{\Gamma(n+1)}{\Gamma\left(\frac{n}{2}+2\right) 2^{n / 2}} X^{n / 2+1} \Delta^{n / 2}\left(\log \frac{1}{\Delta}\right)^{n / 2}+o\left(X^{n / 2+1} \Delta^{n / 2}\left(\log \frac{1}{\Delta}\right)^{n / 2}\right) .
\end{aligned}
$$

Consequently, adding (23) and (24), we have the theorem. 


\section{Numerical evidence}

Instead of having the first main terms only in Theorem 1.1 and 1.2 one should expect more to be true, namely,

Conjecture 4.1. For every $\epsilon>0$ and $\lambda>0$,

$\int_{1}^{X}|\psi(x+h)-\psi(x)-h|^{\lambda} d x=\frac{\Gamma(\lambda+1)}{\Gamma\left(\frac{\lambda}{2}+1\right) 2^{\lambda / 2}} h^{\lambda / 2+1} \int_{E}^{X / h}\left(\log \frac{x}{E}\right)^{\lambda / 2} d x+o\left(X h^{\lambda / 2}\right)$

for $X^{\epsilon} \leq h \leq X^{1-\epsilon}$. Here $E=2 \pi e^{C_{0}-1}$.

Conjecture 4.2. For every $\epsilon>0$ and $\lambda>0$,

$\int_{1}^{X}|\psi(x+\delta x)-\psi(x)-\delta x|^{\lambda} d x=\frac{\Gamma(\lambda+1)}{\Gamma\left(\frac{\lambda}{2}+2\right) 2^{\lambda / 2}} X^{\lambda / 2+1} \delta^{\lambda / 2}\left(\log \frac{1}{E \delta}\right)^{\lambda / 2}+o\left(X^{\lambda / 2+1} \delta^{\lambda / 2}\right)$

for $X^{-1+\epsilon} \leq \delta \leq X^{-\epsilon}$. Again $E=2 \pi e^{C_{0}-1}$.

Using a C program, we get some numerical evidence in support of Conjecture

4.2 as well as the odd moments for (2). Firstly, regarding Conjecture 4.2

For $X=10^{8}$ and $\delta=10^{-4}$ :

$\lambda \quad$ Actual value for $\lambda$-th moment Result from formula

$1.01 .5009 * 10^{10} \quad 1.4851 * 10^{10}$

$2.17 .1441 * 10^{12} \quad 6.9344 * 10^{12}$

$3.2 \quad 4.8737 * 10^{15} \quad 4.6213 * 10^{15}$

$4.34 .1913 * 10^{18} \quad 3.8864 * 10^{18}$

$5.4 \quad 4.2519 * 10^{21} \quad 3.8768 * 10^{21}$

$6.54 .8884 * 10^{24} \quad 4.4213 * 10^{24}$

For $X=10^{10}$ and $\delta=10^{-5}$ :

$\lambda$ Actual value for $\lambda$-th moment Result from formula

$\begin{array}{lll}1.0 & 5.3464 * 10^{12} & 5.3452 * 10^{12}\end{array}$

$\begin{array}{lll}2.1 & 1.0218 * 10^{16} & 1.0210 * 10^{16}\end{array}$

$\begin{array}{lll}3.2 & 2.7871 * 10^{19} & 2.7835 * 10^{19}\end{array}$

$\begin{array}{lll}4.3 & 9.5892 * 10^{22} & 9.5764 * 10^{22}\end{array}$

$\begin{array}{lll}5.4 & 3.9120 * 10^{26} & 3.9079 * 10^{26}\end{array}$

$6.51 .8248 * 10^{30} \quad 1.8232 * 10^{30}$

Note: We just happen to pick some values for $\lambda$.

Secondly, for $n$ odd,

$$
\int_{1}^{X}(\psi(x+\delta x)-\psi(x)-\delta x)^{n} d x=o\left(X^{n / 2+1} \delta^{n / 2}\left(\log \frac{1}{\delta}\right)^{n / 2}\right) .
$$


For $X=10^{8}$ and $\delta=10^{-4}$ :

$\begin{array}{lll}n & \text { Actual value for } n \text {-th moment } & \frac{\Gamma(n+1)}{\Gamma\left(\frac{n}{2}+2\right) 2^{n / 2}} X^{n / 2+1} \delta^{n / 2}\left(\log \frac{1}{\delta}\right)^{n / 2} \\ 1 & -4.9574 * 10^{7} & 1.6143 * 10^{10} \\ 3 & -2.0632 * 10^{13} & 1.7842 * 10^{15} \\ 5 & -3.3174 * 10^{18} & 4.6952 * 10^{20}\end{array}$

For $X=10^{10}$ and $\delta=10^{-5}$ :

$n$ Actual value for $n$-th moment

$17.2371 * 10^{8}$

$\frac{\Gamma(n+1)}{\Gamma\left(\frac{n}{2}+2\right) 2^{n / 2}} X^{n / 2+1} \delta^{n / 2}\left(\log \frac{1}{\delta}\right)^{n / 2}$

$-1.3468 * 10^{16}$

$5.7074 * 10^{12}$

$5-2.5587 * 10^{23}$

$7.8851 * 10^{18}$

$2.5937 * 10^{25}$

Note: $\frac{\Gamma(n+1)}{\Gamma(n / 2+2) 2^{n / 2}}$ acts as a normalization constant coming from the main term of $\lambda$-th moment.

\section{References}

[1] T.H. Chan, Higher moments of primes in short intervals I, preprint.

[2] A. Ghosh. On Riemann zeta-function - sign changes of $S(T)$, Recent Progress in analytic number theory, vol. 1, Academic Press, New York, 1981, pp. 25-46.

[3] A. Ghosh. On the Riemann zeta-function - Mean value theorems and the distribution of $|S(T)|$, J. Number Theory 17, 1983, 93-102.

[4] D.A. Goldston and H.L. Montgomery, On pair correlations of zeros and primes in short intervals, Analytic Number Theory and Diophantine Problems (Stillwater, OK, July 1984), Prog. Math. 70, Birkauser, Boston, 1987, pp. 183-203.

[5] H.L. Montgomery and K. Soundararajan, Primes in Short Intervals, preprint.

[6] H.L. Montgomery and R.C. Vaughan, On the distribution of reduced residues, Annals of Math. 123, 1986, 311-333.

Tsz Ho Chan

American Institute of Mathematics

360 Portage Avenue

Palo Alto, CA 94306

USA

thchan@aimath.org 